ISSN electrónico: 2172-9077

DOI: http://dx.doi.org/10.14201/fjc201613129146

\title{
EL ÍNDICE DE POTENCIALIDAD DE LAS INDUSTRIAS CULTURALES Y CREATIVAS
}

\section{The Cultural and Creative Industry Potential Index}

\section{Dr. Antonio CASTRO-HIGUERAS}

Profesor asociado de Comunicación Audiovisual y Publicidad. Universidad de Málaga, España

E-mail: acastro@uma.es

(iD) http://orcid.org/0000-0001-7406-5550

Dr. Miguel DE AGUILERA MOYANO

Catedrático de Comunicación Audiovisual y Publicidad. Universidad de Málaga, España

E-mail: deaguilera@uma.es

(iD) http://orcid.org/0000-0001-7406-5550

Fecha de recepción del artículo: 21/04/2016

Fecha de aceptación definitiva: 12/09/2016

\begin{abstract}
RESUMEN
Las industrias culturales y creativas se han convertido en un sector estratégico para la nueva economía del conocimiento y, en consecuencia, para las administraciones públicas que promueven el sector a través de sus políticas. En España, la relación entre cultura, creatividad y desarrollo económico se ha ido consolidando en los últimos años hasta alcanzar un 3,5\% del PIB y un 3\% del empleo total en 2014. La importancia del sector requiere de un diagnóstico que facilite la toma de decisiones. Para cubrir esta necesidad han proliferado sistemas de indicadores cuyo objetivo es la evaluación de la creatividad en un territorio determinado. En el presente artículo se analizan y revisan los distintos índices existentes para, a partir de estos, elaborar un indicador sintético: el Índice de potencialidad de las industrias culturales y creativas, que aborda esta realidad a partir de tres dominios: base cultural, políticas públicas e industrias culturales y creativas.
\end{abstract}

Palabras clave: Industrias culturales y creativas; creatividad; índice; políticas culturales; ciudades creativas.

\begin{abstract}
The cultural and creative industries are regarded as a strategic sector in today's knowledge economy and are therefore encouraged by government policies. The link between culture, creativity and economic development has flourished in Spain in recent years, to the extent that by 2014 the sector accounted for 3.5\% of the country's GDP and $3 \%$ of all jobs. Such an important sector requires a diagnostic tool to support decision making. A number of indicator systems have been put forward to meet this need, all of which are designed to evaluate creativity in a given field. This article examines and reviews the various indicators that have been proposed and uses them to develop a composite indicator - the Cultural and Creative Industry Potential Index - which measures the sector's potential from three angles: the cultural base, government policies, and the cultural and creative industries themselves.
\end{abstract}

Key words: Creative and Cultural Industries; Creativity; Cultural Policies; Creative Cities. 


\section{INTRODUCCIÓN}

La concepción de la cultura como generadora de actividad económica ha sido ampliamente estudiada, sobre todo, desde la década de los sesenta. Desde que se acuñó la expresión «industrias creativas», su estudio ha aumentado exponencialmente, trascendiendo el ámbito académico y científico para adentrarse en el campo institucional mediante las políticas públicas. El reconocimiento de la importancia de la creatividad para el desarrollo económico ha calado en las instituciones europeas, nacionales, regionales y locales. Las industrias culturales y creativas se han convertido, pues, en un sector estratégico para las administraciones públicas en relación con la nueva economía del conocimiento.

Y ello ha exigido conocer y comprender cómo funcionan estas industrias, pero también establecer procedimientos para su precisa medición, dando una base seria para la formulación de políticas, la toma de decisiones y la evaluación de la eficacia de las políticas públicas en este área.

Un problema inicial que se presenta al abordar el sector es el de su misma definición. Aun sin existir una delimitación unánime de las industrias culturales y/o creativas, se ha descrito el peso que ocupa el sector en la economía nacional, regional y, en menor medida, local. En el caso de España, la relación entre cultura, creatividad y desarrollo económico se ha ido consolidando en los últimos años, como nos muestran los datos de 2014, donde el sector cultural aportó el 3,5\% del PIB y supuso 511.800 puestos de trabajo, un 3\% del empleo total con un 3,5\% del total del tejido empresarial, conformándose las ciudades como verdaderos núcleos de este sector económico (Ministerio de Educación, Cultura y Deporte, 2015).

A pesar de conocerse estos datos, son sorprendentemente escasos los estudios relacionados con la industria cultural y creativa de nuestro país en el ámbito que nos preocupa en este estudio: la evaluación y dimensionamiento del sector y, en particular, en el ámbito geográfico local, concretamente el urbano.

\section{LOS ÍNDICES COMO HERRAMIENTA EVALUADORA DE LA CREATIVIDAD}

Operacionalmente, los índices o grupos de indicadores proporcionan una aproximación a un fenómeno complejo y multidimensional, no medible de manera directa. Los indicadores, por su parte, son «los correlatos empíricos de las variables que se intentan medir, son sus expresiones concretas, prácticas, medibles» (Batthyány, et al., 2011, p. 57). En la medición de los procesos sociales los índices constituyen una poderosa herramienta de tipo cuantitativo para el estudio de realidades complejas y la posterior toma de decisiones en cuanto a la definición de políticas públicas. A pesar de las imprecisiones inherentes a todo sistema para capturar la realidad, pues se trata del establecimiento de una correspondencia entre el «mundo real» y el «mundo conceptual» desde la perspectiva del investigador «no hay medición perfecta» (Hernández Sampieri, Fernández Collado y Baptista Lucio, 2010, p. 200)-, el instrumento de medición idóneo, en nuestro caso un sistema de indicadores, es el que se acerca fielmente a la representación de las variables observadas (Hernández Sampieri, Fernández Collado y Baptista Lucio, 2010).

Esta herramienta analítica es utilizada por gobiernos e instituciones, nacionales y supranacionales, para medir, monitorizar y conocer las distintas realidades sociales, económicas, etc. En esta línea, son significativos ejemplos el Science, Technology and Industry Scoreboard (OCDE, 2013), que analiza las tendencias de la economía del conocimiento a nivel global o, en el escenario europeo, el Panel de Indicadores de Innovación o Innovation Union Scoreboard de la Comisión Europea (2015), que mide y evalúa la innovación en los distintos países y regiones de la Unión Europea.

En el caso de la creatividad, sus relaciones con el desarrollo económico están siendo objeto de debate en el ámbito académico y político. Esta actividad investigadora ha favorecido la proliferación

(C) Ediciones Universidad de Salamanca / CC BY - NC ND Fonseca, Journal of Communication, n. 13, 2016, pp. 129-146 
de índices que evalúan la creatividad de un entorno local, regional o nacional. Como muestra más destacada encontramos el Creativity Scoreboard de la Unión Europea, actualmente en fase de estudio a partir de la propuesta del Informe KEA (Comisión Europea, 2009).

Si nos retrotraemos a la aparición de esta necesidad evaluadora de la creatividad aplicada a un territorio, hemos de señalar que los primeros índices de creatividad aparecieron a principios del presente siglo, coincidiendo con la distinción, y auge paralelo, de las industrias creativas. Richard Florida (2002) fue uno de los primeros en diseñar y aplicar un índice para ciudades creativas. La principal novedad de su aportación consistió en la inclusión de indicadores no habituales en índices similares -como los de innovación, donde predominan los indicadores de producción industrial-, mientras que Florida incorpora nuevos indicadores de carácter personal y social como el talento, la apertura, tolerancia, diversidad, habitabilidad, etc. Con el modelo de Florida como referencia comienzan a proliferar índices que abordan la creatividad con enfoques diferentes, si bien ejerciendo sobre la mayoría de ellos una gran influencia el Creativity Cities Index de Richard Florida. Y nosotros, por nuestra parte, queremos contribuir a ello mediante la elaboración de un índice de creatividad, denominado «Índice de potencialidad de las industrias culturales y creativas», partiendo de la hipótesis de que la realidad analizada en la revisión de los índices existentes se puede agrupar y aislar en tres estructuras superiores interrelacionadas entre sí pero independientes: la base cultural y creativa, la política cultural y los resultados de las industrias culturales y creativas.

\section{3. ОВJETIVOS}

El primer objetivo de la investigación es la creación del Índice de potencialidad de las industrias culturales y creativas. Esta herramienta, que permitirá cubrir el vacío de conocimiento mencionado en el apartado anterior, se construye sobre todo a partir de la revisión, análisis y síntesis de los distintos indicadores y dimensiones presentes en los índices pre-existentes, elaborándose una propuesta novedosa y avanzada que validamos con distintos procedimientos. Este índice que proponemos no se limita, por supuesto, a un mero ejercicio de síntesis de los anteriores; antes bien, hemos de señalar que su novedad también viene dada por el enfoque adoptado. Mientras que la mayoría de índices se organizan en dimensiones, en el sistema de indicadores propuesto en este artículo se determinan unas estructuras superiores o dominios que agrupan estas dimensiones en función de la base cultural y creativa, la política cultural y los resultados de las industrias culturales y creativas.

El segundo objetivo se deriva del anterior y busca la aplicación del Índice de potencialidad de las industrias culturales y creativas a la medición de la capacidad de desarrollo económico del sector en un territorio concreto, determinado a partir de los vectores o dominios ya mencionados y con la finalidad última de servir de herramienta para el diseño de políticas públicas adecuadas y oportunas.

\section{Metodología}

Para la construcción del índice hemos utilizado un procedimiento consistente, primero, en el análisis y síntesis de los índices de creatividad existentes, la puesta en común de las dimensiones más repetidas y su agrupación en los dominios propuestos.

Una vez desarrollada la propuesta de índice, y con el fin de preservar uno de los principios que rigen la construcción de sistemas de indicadores como es la adaptación a la realidad evaluada, así como de completar nuestra propuesta y validarla en su caso, utilizamos un procedimiento basado en una de las técnicas de análisis social que entre otras cuestiones permite su adecuación a las condiciones particulares del territorio evaluado: el método Delphi o panel de expertos (Landeta, Matey, Ruíz y Villareal, 2002; Konow y Pérez, 1990). Esta técnica de investigación cualitativa de carácter exploratorio, que 
busca el consenso grupal, ha pues de validar y consensuar los distintos indicadores a través de un grupo de expertos con perfiles multidisciplinares, procedentes del ámbito académico, la industria, el emprendimiento, la creación, el análisis sociológico, la gestión cultural o la política. En este proceso abierto, los expertos pueden incorporar nuevas propuestas de indicadores que completen y mejoren el índice.

Los datos obtenidos de la explotación del índice, una vez finalizado el proceso de discusión y establecido el índice definitivo, pasan por un proceso de normalización para la obtención de valores independientemente de la unidad de medida utilizada en cada uno de los indicadores. El método de normalización empleado es z-scores (Nardo, Saisana, Saltelli, Tarantola, Giovannini y Hoffmann, 2008). Esta medida del grado de dispersión centra y reduce las variables en torno de cero a partir de la media de los valores obtenidos tras la medición y su desviación típica o estándar.

A su vez, la ponderación de pesos o importancia relativa de cada indicador y dimensión en el total del índice es siempre la fase más discutida, por tratarse de juicios de valores, en el proceso de elaboración de este tipo de índices (Nardo, et al., 2008). En la aplicación del Índice de potencialidad de las industrias culturales y creativas proponemos tanto la utilización de la ponderación de pesos igualitarios (o equal weigths), en la que todos los indicadores tienen el mismo valor. Por último, utilizamos el método de agregación aditivo (Nardo, et al., 2008), que realiza la media aritmética de los valores de cada indicador en cada una de las dimensiones o dominios para, finalmente, obtener el resultado total del índice.

El criterio utilizado en las distintas fases de la construcción del índice, normalización, ponderación y agregación, es la simplicidad metodológica. Otros autores también optan por esta en sus evaluaciones debido a la complejidad antes mencionada (Florida; Picard, Gronlud y Toivonen; KEA; Hollanders y Van Cruysen; Hartley, etc.). Se trata, pues, de construir un instrumento para el análisis, más que de presentar un mero ranking clasificatorio compuesto por las sumas de los indicadores.

\section{RESUltados}

En primer lugar, analizamos los distintos índices utilizando como estructura para su presentación la tipología de Hartley (2012), en la que distingue entre: (1) índices basados en la cultura y la creatividad (culture-based indexes), (2) índices de ciudades globales, redes y tecnología (technology-based indexes) y (3) otras aproximaciones, siendo el primer grupo el que suscita mayor interés en nuestro estudio. Tras examinar en profundidad los dos principales grupos se observa que los índices de base cultural miden en especial la vitalidad del sector creativo en un entorno determinado a partir de su producción, empleo, participación y talento, siendo determinante el talento y el capital humano para el desarrollo de una ciudad. En cambio, los índices de base tecnológica incluyen los indicadores creativos como un apartado más junto a otras categorías como el medio ambiente, transporte, tecnología, etc. Este enfoque, más amplio, se centra en las infraestructuras e interconexiones de una ciudad en sus diferentes niveles.

\section{1. ÍNDICES DE BASE CULTURAL}

\subsubsection{CREATIVE Cities INDEX DE RiCHARD FLORIDA}

Como ya se ha señalado, el experto en geografía económica Richard Florida propuso un índice como herramienta para evaluar y clasificar la potencialidad creativa de las regiones metropolitanas en su obra The Rise of Creative Class (Florida, 2002). 
En su argumentación sobre la clase creativa, el autor sostiene que «las ciudades con mayor número de artistas, músicos, profesores y científicos, trabajadores tecnológicos, extranjeros, comunidad gay y los llamados bohemios tendrán mayores niveles de desarrollo económico» (Florida, 2002, p. 12). En esta obra reprocha a las autoridades que pongan el foco en la atracción de inversiones y empresas en lugar de invertir en la atracción de la clase creativa, y propone un índice que capta y evalúa esa realidad. Florida agrupa las dimensiones en torno de tres conceptos a los que denomina las 3T: Talento, Tecnología y Tolerancia.

En cuanto a lo metodológico, estos tres subíndices tienen el mismo peso para la configuración del resultado final. Las fuentes de datos utilizadas proceden de otros índices y datos oficiales, la mayor parte de carácter cuantitativo - excepto los referidos a la tolerancia-, procedentes de encuestas.

El Creative Cities Index ha sido adaptado a diferentes regiones y territorios por numerosos autores (Marlet y Van Woerkens, 2004) (Boschma \& Fritsch, 2009), incluido el propio Florida que, junto a Irene Tinagli, construyó el Euro-Creativity Index (Florida y Tinagli, 2004). El autor, director del Martin Prosperity Institute de Toronto, ha desarrollado asimismo a partir de su índice de las 3T el Global Creativity Index (Florida, Mellander, Stolarick, Sik, Matheson y Hopgood, 2011) que, con algunas modificaciones, permite analizar y clasificar en ranking de creatividad los diversos países.

En el plano conceptual, se ha llegado incluso a ampliar el índice de Florida añadiendo una 4T, de territorio. Acs y Zegyesi (2009), plantean en su estudio de la ciudad de Baltimore esta cuarta dimensión o subíndice, en la que incluyen indicadores como índices de desigualdad salarial, índices de inaccesibilidad a la vivienda y estadísticas sobre el coste de la vivienda.

La obra de Florida ha tenido una gran popularidad y repercusión en el ámbito académico e institucional, tanto por su condición pionera como, sobre todo, por su heterodoxa e innovadora visión de la creatividad aplicada al desarrollo económico de un territorio.

Su Creative Cities Index ejerce una gran influencia sobre los índices posteriormente elaborados, que han llegado a calificarse como índices floridianos (Hartley, 2012). Pero, por otro lado, su índice ha recibido también numerosas críticas de diversos expertos, que le achacan cuestiones tales como la falta de rigor metodológico (Peck, 2005), la inclusión de la tolerancia como factor que genera crecimiento económico (Glaeser y Saiz, 2004) o la poca importancia de los atractivos urbanos para la localización del talento (Clark, 2004).

\subsubsection{Cultural Life INDEX}

Este índice, promovido por el Ministerio de Educación y Cultura finlandés (Picard, Gronlud y Toivonen, 2003), tiene por objetivo la creación de un sistema de indicadores transversal a los distintos sectores a partir de datos extraídos de fuentes estadísticas oficiales como Eurostat o UNESCO. Con un ámbito de aplicación nacional, el Cultural Life Index parte de la base de que un ambiente cultural rico beneficia la creatividad y, por otro lado, la vida social que promueven las actividades sociales apoyan la economía creativa.

Una de las aportaciones de este índice es su estructuración en tres vectores que interactúan en el ecosistema cultural: la oferta o disponibilidad cultural, la demanda o participación cultural y la actividad cultural generada o producción cultural.

El Cultural Life Index no es un índice propiamente dicho sino, más bien, una propuesta para su discusión que, aunque no ha sido llevada a la práctica, pone sin embargo de manifiesto una visión más cultural y social y menos económica. 


\subsubsection{Hong Kong CREATIVITY INDEX}

Este índice, creado en 2004 por el Centre for Cultural Policy Research de la Universidad de Hong Kong (Hui, Ng, Mok, Ngai, Wan-kan y Yuen, 2004), recibe una gran influencia del índice de Richard Florida. Los autores parten de la premisa de que «un acto creativo puede analizarse aplicando el concepto de ciclo de actividad creativa» (Hui, et al., 2004, p. 40). Según los autores, la capacidad de crear se inserta en el contexto de tres tipos o formas de capital: el capital social, el capital cultural y el capital humano; mientras que la articulación para su promoción viene dada por facilitadores sociales, institucionales y de mercado, a los que se refieren como capital estructural e institucional. Por último, los efectos producidos por la interrelación de estas formas de capital se expresan en los resultados de la creatividad.

El Hong Kong Creativity Index tiene una finalidad de alcance exclusivamente local, ya que resulta del análisis de la ciudad de Honk Kong durante los años 1999 a 2004. A nivel metodológico, los resultados del índice están validados estadísticamente por el Análisis de Componentes Principales (ACP) y normalizados con referencia a 100 .

Este modelo, derivado del índice de las 3T de Florida, profundiza en los resultados económicos y tecnológicos de la creatividad. Entre los aspectos evaluados reseñables destacan las infraestructuras culturales o la participación social. Cabe destacar la incorporación de elementos de competitividad como los presentes en la encuesta Global Competitiveness Report (Schwab y Sala-i-Martin, 2011).

La novedad y aportación de este índice radica en la inclusión de los outputs o resultados de las industrias creativas como dimensión a tener en cuenta por su poder de realimentación del sistema económico creativo, además de la incorporación de las políticas públicas como elemento del capital institucional.

\subsubsection{COMPOSITE INDEX OF THE CREATIVE ECONOMY}

El índice elaborado en 2008 por los académicos Harry Bower, de la Universidad de Queens de Carolina del Norte, y Wim Moesen y Leo Sleuwaegen, de la Universidad Católica de Lovaina (Bowen, Moesen \& Sleuwaegen, 2008), analiza la capacidad creativa de regiones europeas a través de los logros en innovación, emprendimiento y apertura, combinando los indicadores clásicos de Florida junto con otros específicos de actividad empresarial o de infraestructuras de tecnologías de la comunicación.

Las fuentes utilizadas se basan en su mayor parte en datos estadísticos de tipo cuantitativo. Una valiosa aportación metodológica frente a otros índices que realizan una simple agregación de valores es la ponderación de las distintas subdimensiones a partir del Análisis Envolvente de Datos (DEA).

\subsubsection{EuROPEAN CREATIVITY INDEX}

Es la propuesta que presenta la consultoría KEA European Affairs en 2009, por encargo de la Comisión Europea, con el fin de promover un índice que evalúe la creatividad en el ámbito de las naciones y regiones europeas (Comisión Europea, 2009). El European Creativity Index también se basa en gran parte en el índice de Florida, y está compuesto por 6 pilares y 32 indicadores, incluidos los resultados de la creatividad que se consolidan como elementos a evaluar tras su incorporación al Honk Kong Creativity Index.

La propuesta de índice para la Unión Europea no se ha llevado a la práctica y continúa en proceso de discusión. Para nuestra propuesta es interesante la estructura de sus dimensiones, aunque los indicadores que la componen son de difícil aplicación a ciudades, por su orientación a la evaluación de las regiones y países europeos. 


\subsubsection{Design, CREATIVITY AND INNOVATION SCOREBOARD}

El Design, Creativity and Innovation Scoreboard (Hollanders y Van Cruysen, 2009) está basado en el Marcador Europeo de Innovación (Comisión Europea, 2009), estudio realizado por el propio Hollanders para la Unión Europea desde la Universidad de Maastricht. Su principal aportación es la conexión que hace entre la innovación, el diseño y la creatividad a partir de un modelo que distingue entre ambiente creativo y creatividad y diseño que, a su vez, conecta con la innovación.

Este índice, compuesto por 35 indicadores agrupados en 7 dimensiones, muestra asimismo influencias floridianas. La novedad radica en la incorporación y evaluación de las actividades de diseño, algo inusual en los índices de creatividad existentes.

En el plano metodológico, cada una de las 7 dimensiones se normalizan utilizando el método de máximo-mínimo. En cuanto a la ponderación de indicadores y dimensiones utiliza la técnica de pesos igualitarios o equal weight.

\subsubsection{CREATIVE City INDEX}

Creado por el Centre of Excellence for Creative Industries and Innovation de la Universidad de Queensland (Australia), con John Hartley como principal autor. Su principal hipótesis es «que para convertirse en una ciudad global es necesario ser antes una ciudad creativa» (Hartley, et al., 2012, p. 47).

El índice está compuesto por 74 indicadores agrupados en 8 dimensiones. Las principales novedades son la incorporación de dimensiones como la economía de la atención y la microproductividad o productividad en la red. Hartley ha sido el primero en valorar la actividad digital como elemento facilitador de la actividad creativa.

A nivel metodológico, el CCI Creative Cities Index utiliza la no ponderación con un peso igual para todos los indicadores y dimensiones; en cuanto a la normalización utiliza la referencia a máximos donde el mayor valor se toma como la puntuación más alta posible.

\section{2. ÍNDICES DE BASE TECNOLÓGICA Y CIUDADES GLOBALES}

Este tipo de índices pone su foco en la economía y tecnología, dejando de lado los aspectos más sociales y culturales. Este hecho, unido a que buena parte de estos índices están diseñados para evaluar entornos metropolitanos muy amplios (como las grandes ciudades que proliferan en nuestros días), hace que no sean de especial interés para nuestra investigación. A pesar de ello nos parece interesante describir los aspectos más significativos de los principales sistemas de indicadores.

The Global Cities Index, realizado por la consultora A. T. Kearney (2014) para la revista económica Foreing Policy y publicado bianualmente desde 2008, evalúa y clasifica 84 ciudades globales y emergentes de todo el mundo, utilizando 26 indicadores agrupados en 5 dimensiones, referidos a la actividad económica, el capital humano, el intercambio de información, la experiencia cultural y el compromiso político. El análisis de las ciudades y su evolución en el tiempo, gracias a las cuatro ediciones publicadas, constituye uno de los mayores atractivos del índice y ha permitido extraer interesantes conclusiones como la tendencia de las ciudades analizadas hacia una mayor globalización.

El Global Power City Index, publicado desde 2008 por la Mori Memorial Foundation (2014), evalúa y clasifica las principales ciudades del mundo en función de su poder para atraer talento y empresas. El índice, dirigido por los prestigiosos académicos Peter Hall y Saska Sassen, está compuesto por 6 dimensiones y 69 indicadores. Su principal aportación radica en su aplicación desagregada por acto- 
res de la ciudad, asignando valores en cada dimensión según se trate de directivos de empresas, investigadores, creadores, visitantes o residentes.

The Shift Index es un índice económico y tecnológico de ciudades y regiones realizado por la consultora Deloitte (Hagel, Brown y Davidson, 2009). Compuesto por 3 subíndices y 25 indicadores, incorpora indicadores de largo plazo que permiten evaluar el cambio que la tecnología y las políticas públicas están provocando en la economía. La principal aportación de este índice es fijarse sobre todo en la evaluación de los cambios. También es interesante su estructura y división en dominios o subíndices relacionados con la base, la actividad y los resultados.

\subsection{OTROS ÍNDICES}

A los índices anteriormente descritos, y agrupados con base en sus enfoques cultural o tecnológico, podemos incorporar otros sistemas de indicadores como el Creative City Index, el Creative Grid o los indicadores de la UNESCO o la UNCTAD.

Charles Landry, quien acuñó el término ciudad creativa, construyó asimismo un índice de creatividad denominado «Landry's Index» para la ciudad de Bilbao, que posteriormente ha sido también aplicado a otras ciudades (Landry, 2010). De este trabajo de campo extrajo, dos años más tarde, la obra The Creative City Index: Measuring the pulse of the city (Landry y Hyams, 2012). Landry distingue 10 dominios que van desde el marco político, el talento o el desarrollo urbano. El índice utiliza principalmente técnicas cuantitativas como encuestas online masivas, sesiones grupales o entrevistas. Al tratarse de consultoría, la metodología utilizada no es clara, por lo que no se puede definir estrictamente como un índice.

Otro caso a reseñar es el Creativity Grid que, más que un índice, es un conjunto de líneas de actuación para orientar las políticas públicas del sector creativo. Se deriva del informe de la DCMS Creative Economy Programme: Infraestructure Working Group (DCMS, 2010). En él se detallan las diez condiciones relativas a infraestructuras necesarias para el crecimiento y la competitividad de las industrias creativas.

En tercer lugar, la Guía para el desarrollo de las industrias culturales y creativas (UNESCO, 2010) define indicadores para el diagnóstico del sector cultural y creativo. Al igual que los anteriores, no se trata de un índice formal, sino de una propuesta de indicadores, a modo de ejemplo, para que se adapten a la realidad específica que se necesite evaluar. La propuesta está estructurada en ocho dimensiones relacionadas con la oferta y la demanda, el empleo o la acción gubernamental.

Por último, el Informe de la economía creativa (UNCTAD, 2013) distingue entre recursos culturales disponibles en una ciudad o región, infraestructuras para el desarrollo de estos recursos y resultados de la creatividad. Esta propuesta de la UNCTAD pretende ser una herramienta para la evaluación y el diagnóstico de la economía creativa local y el posterior diseño e implementación de políticas públicas.

Como hemos podido comprobar, la mayor parte de los índices han sido creados para evaluar ciudades o regiones, mientras que algunos otros han sido diseñados como una herramienta válida para el estudio de la creatividad aplicada a la economía en un territorio determinado, pero en todos los casos la finalidad última es la de servir de herramienta para la optimización de las políticas públicas, de modo que contribuyan al desarrollo y al crecimiento económico del territorio. Pero esta realidad social y económica que se pretende evaluar no puede escapar a la mirada crítica. En la evolución de las industrias culturales y creativas subyace un debate ideológico en el que autores notables autores, como Zallo (1988) u otros, les otorgan una función de reproducción social e ideológica. El «mero economicismo» (Schlesinger, 2009; Bustamante, 2011; Zallo, 2011) o su «carácter neoliberal» (Miller, 2009; Bustaman- 
te, 2011) son los argumentos más utilizados por los autores críticos con esta visión de la cultura y la creatividad.

Pero volviendo al plano metodológico, podemos observar que la mayor parte de los índices utilizan la no ponderación en sus resultados, es decir, la importancia relativa o asignación de pesos a cada indicador o dimensión son iguales; en cambio otros, como el Composite Index of the Creative Economy, utilizan técnicas de ponderación endógenas (Bowen, Moesen y Sleuwaegen, 2008). En cuanto a la normalización de los datos, algunos índices utilizan técnicas como el método de normalización mínimo-máximo que asigna valores entre 0 y 1 (Oliveira, 2012) o el método de referencia a máximos (Hartley, et al., 2012).

En las siguientes tablas se muestran los distintos índices a través de las dimensiones que los componen.

Tabla 1. Cuadro comparativo de dimensiones e índices

\begin{tabular}{|c|c|c|c|c|c|c|c|}
\hline & $\begin{array}{l}\text { Creative } \\
\text { Cities } \\
\text { Index }\end{array}$ & $\begin{array}{l}\text { Cultural } \\
\text { Llfe } \\
\text { Index }\end{array}$ & $\begin{array}{l}\text { Hong Kong } \\
\text { Creativity } \\
\text { Index }\end{array}$ & $\begin{array}{c}\text { Composite } \\
\text { Index }\end{array}$ & $\begin{array}{c}\text { European } \\
\text { Creativity } \\
\text { Index }\end{array}$ & $\begin{array}{c}\text { Design, } \\
\text { Creativity } \\
\& \\
\text { Innovation } \\
\end{array}$ & $\begin{array}{c}\text { CCI } \\
\text { Creative } \\
\text { City } \\
\text { Index }\end{array}$ \\
\hline Educación & $\square$ & & $\square$ & $\square$ & $\square$ & $\square$ & $\square$ \\
\hline Capital humano & $\square$ & & $\square$ & & $\square$ & & $\square$ \\
\hline Diversidad & $\square$ & & $\square$ & $\square$ & $\square$ & $\square$ & $\square$ \\
\hline Tolerancia & $\square$ & & $\square$ & & $\square$ & $\square$ & $\square$ \\
\hline $\begin{array}{l}\text { Ambiente cívico/ } \\
\text { Compromiso } \\
\text { social }\end{array}$ & & & $\square$ & & $\square$ & & $\square$ \\
\hline Oferta cultural & & $\square$ & $\square$ & & 四 & & $\square$ \\
\hline Demanda cultural & & $\square$ & $\square$ & & $\square$ & $\square$ & $\square$ \\
\hline $\begin{array}{l}\text { Producción } \\
\text { cultural }\end{array}$ & & $\square$ & $\square$ & & $\square$ & & \\
\hline Tecnología & $\square$ & $\square$ & $\square$ & $\square$ & 田 & & $\square$ \\
\hline $\mathrm{I}+\mathrm{D}+\mathrm{i}$ & $\square$ & & $\square$ & $\square$ & & $\square$ & $\square$ \\
\hline Competitividad & & & & & & $\square$ & \\
\hline $\begin{array}{l}\text { Emprendimiento / } \\
\text { empresa }\end{array}$ & & & $\square$ & $\nabla$ & & & $\square$ \\
\hline Empleo & $\square$ & & $\square$ & & $\square$ & $\square$ & $\square$ \\
\hline $\begin{array}{l}\text { Resultados } \\
\text { económicos }\end{array}$ & & & $\square$ & & $\square$ & $\square$ & $\square$ \\
\hline Políticas públicas & & & $\square$ & & $\square$ & & $\square$ \\
\hline Financiación & & & $\square$ & (7) & 口 & & $\square$ \\
\hline $\begin{array}{l}\text { Productividad en } \\
\text { la red }\end{array}$ & & $\nabla$ & & & & & $\square$ \\
\hline $\begin{array}{l}\text { Conectividad } \\
\text { virtual }\end{array}$ & & $\square$ & & & & & $\square$ \\
\hline $\begin{array}{l}\text { Accesibilidad / } \\
\text { comunicaciones }\end{array}$ & & & & & & & $\square$ \\
\hline Infraestructuras & & & $\square$ & & & & $\square$ \\
\hline Flujo de personas & & & $\square$ & & & & $\square$ \\
\hline Habitabilidad & & & $\square$ & & & & \\
\hline $\begin{array}{l}\text { Medio natural - } \\
\text { urbano }\end{array}$ & $\square$ & & & & & $\square$ & \\
\hline
\end{tabular}

Fuente: Elaboración propia a partir de las fuentes consultadas. 
Tabla 2. Dimensiones o temas más comunes en los índices analizados

\begin{tabular}{|c|c|}
\hline Dimensiones o temas & $\mathrm{N}^{\circ}$ índices en los que aparece \\
\hline Educación & 6 \\
\hline Tecnología & 6 \\
\hline Diversidad & 6 \\
\hline Empleo & 5 \\
\hline Demanda cultural & 5 \\
\hline Tolerancia & 5 \\
\hline $\mathrm{I}+\mathrm{D}+\mathrm{i}$ & 5 \\
\hline Oferta cultural & 4 \\
\hline Capital humano & 4 \\
\hline Resultados económicos & 4 \\
\hline Financiación & 4 \\
\hline Producción cultural & 3 \\
\hline Políticas públicas & 3 \\
\hline Ambiente cívico/ Compromiso social & 3 \\
\hline Emprendimiento/ empresa & 3 \\
\hline Conectividad virtual & 2 \\
\hline Productividad en la red & 2 \\
\hline Medio natural - urbano & 2 \\
\hline Infraestructuras & 2 \\
\hline Flujo de personas & 2 \\
\hline Competitividad & 1 \\
\hline Habitabilidad & 1 \\
\hline Accesibilidad / comunicaciones & 1 \\
\hline
\end{tabular}

Fuente: Elaboración propia a partir de las fuentes consultadas.

\section{El Índice de Potencialidad De las Industrias Culturales Y Creativas}

El resultado de este trabajo de investigación es un índice compuesto por 56 indicadores agrupados en 14 dimensiones que, a su vez, se distribuyen en 3 dominios (Gráfico 1).

Gráfico 1. Dominios del índice de potencialidad de las industrias culturales y creativas

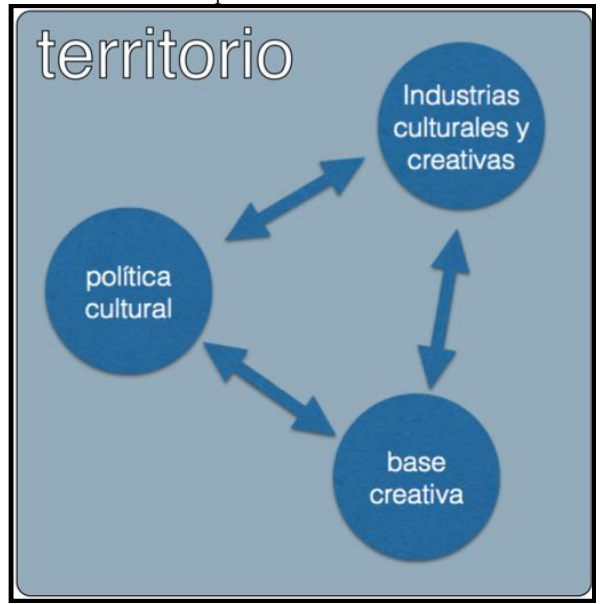

Fuente: Elaboración propia

(C) Ediciones Universidad de Salamanca / CC BY - NC ND $\quad$ Fonseca, Journal of Communication, n. 13, 2016, pp. 129-146 


\subsection{BASE CULTURAL Y CREATIVA}

Es el conjunto de elementos de carácter personal, social, cultural, tecnológico, económico, etc., que potencialmente puedan favorecer la creación, proliferación o desarrollo de las industrias culturales y creativas. Estos componentes, que están relacionados con la creatividad, el talento y el conocimiento, se operacionalizan en este primer dominio del índice.

Esta visión es totalmente nueva respecto de los índices previamente existentes; la idea que más se acerca a ella son los cimientos, o foundation, que aparecen en The Shift Index refiriéndose a las bases para el cambio económico y tecnológico de las ciudades (Hagel, Brown y Davidson, 2009).

El dominio está compuesto por 8 dimensiones. La primera, «población y economía», se refiere a elementos significativos de la realidad demográfica y económica del ámbito geográfico de estudio. Según las tesis de Richard Florida (2002), la concentración poblacional es una característica del entorno creativo por lo que se entiende que una alta concentración de población favorecerá las actividades culturales y creativas.

La dimensión «educación» pone el foco en la enseñanza relacionada con la cultura y la creatividad como motor de desarrollo del talento dentro del sector cultural y creativo. Está compuesta por indicadores que aportan información sobre la oferta educativa y formativa oficial o reglada de ciclos superiores, en su mayoría de carácter público.

Las tecnologías de la información y de la comunicación están reflejadas en la dimensión «tecnología». Las políticas públicas de promoción y apoyo al desarrollo de las TIC denotan la importancia de estas, como se puede comprobar en documentos como la Agenda Digital para Europa (Comisión Europea, 2010) o la Agenda Digital para España (Gobierno de España, 2013). Indicadores referidos a la tecnología y las infraestructuras TIC aparecen en la mayor parte de índices existentes; de hecho, constituyen uno de los tres pilares del índice de Richard Florida de las 3 T (Talento, Tecnología y Tolerancia).

La cuarta dimensión recoge indicadores relacionados con la «tolerancia, diversidad y apertura». Numerosas investigaciones, particularmente en el campo de la economía y del urbanismo, hacen referencia a los vínculos existentes entre apertura y diversidad de una determinada sociedad y creación e innovación. Desde la urbanista Jane Jacobs (1961), pionera en proponer la diversidad y el intercambio de ideas como fuente de innovación en las ciudades, hasta Richard Florida (2002), que relaciona ambiente creativo y conceptos como diversidad, tolerancia y apertura, llegando a concluir que un entorno tolerante atrae al talento creativo y este al capital promoviendo el crecimiento económico, numerosos investigadores relacionan estas componentes de la sociedad con el grado de creatividad.

Por otro lado, en el desarrollo del talento intervienen elementos personales y ambientales que promueven un desempeño superior en uno o más campos de la actividad humana. En este contexto, la dimensión «talento» agrupa los indicadores en torno de categorías como capital humano, producción científica, I+D y emprendimiento, propiedad industrial, propiedad intelectual y premios. Estas subdimensiones se repiten en los índices vistos anteriormente, sobre todo los relacionados con investigación y creación de conocimiento.

La dimensión «producción cultural y creativa» se relaciona con la oferta y la demanda. Estos procesos son ampliamente analizados en índices como el Cultural Life Index, donde aparecen como uno de sus tres pilares junto a la disponibilidad y la participación cultural (Picard, Gronlud y Toivonen, 2003).

En séptimo lugar, la dimensión «experiencia cultural» hace referencia a la interacción que se lleva a cabo entre los diferentes actores culturales. Bajo este epígrafe se agrupan indicadores relacionados con la disponibilidad y la participación cultural. En el primer caso, la disponibilidad se refiere a las 
acciones culturales significativas llevadas a cabo en la ciudad. Respecto de la participación cultural, distinguimos entre consumo cultural, en términos meramente económicos, y las interrelaciones sociales en torno de la cultura, como por ejemplo el asociacionismo cultural. Esta dimensión está influida por el ambiente cultural del European Creativity Index (Comisión Europea, 2006), aunque se sustituye por el término disponibilidad procedente del Cultural Life Index (Picard, Gronlud y Toivonen, 2003).

Por último, en la dimensión «microproductividad» se agrupan indicadores que evalúan la creación de contenidos por parte de los usuarios y su producción e intercambio de información en el ámbito digital. Se trata de conocer el volumen de creación de contenidos generados por los usuarios (usergenerated content) como expresión característica de la Web 2.0, las redes sociales y otras plataformas tecnológicas que favorecen aspectos colaborativos dentro de la producción de contenidos. En esta dimensión se diferencian dos grupos de indicadores, los referidos a usuarios o prosumidores de un ámbito geográfico determinado con carácter general, y los usuarios microproductores relacionados con el sector cultural y creativo. La inclusión de estos indicadores no ha sido habitual en los índices de creatividad, solo el CCI Creative City Index (Hartley, et al., 2012) evalúa la producción realizada por los usuarios en la red, a excepción del número de usuarios de internet que analiza el Cultural Life Index (Picard, Gronlud y Toivonen, 2003). 
ANTONIO CASTRO-HIGUERAS, MIGUEL DE AGUILERA MOYANO

EL ÍNDICE DE POTENCIALIDAD DE LAS INDUSTRIAS CULTURALES Y CREATIVAS

Tabla 3. Dominio Base Cultural y Creativa

\begin{tabular}{|c|c|}
\hline Indicador 1 & Densidad de población \\
\hline Indicador 2 & Coste de la vida \\
\hline \multicolumn{2}{|c|}{ A2. DIMENSION "EDUCACION" } \\
\hline \multicolumn{2}{|c|}{ Subdimensión: Educación universitaria relacionada con la cultura y la creatividad } \\
\hline Indicador 3 & Nümero de titulaciones $y$ plazas oferta das \\
\hline \multicolumn{2}{|c|}{ Subdimensión: Forma dón profesional relacionada con la cultura y la ceatividad } \\
\hline Indicador 4 & Nümero de titulaciones $y$ plazas ofertadas \\
\hline \multicolumn{2}{|c|}{ Subdimensión: Otras enseñanzas superiores regla das relacionadas con la cultura y la creatividad } \\
\hline Indicador 5 & Número de titulaciones de grado superior y plazas ofertadas \\
\hline \multicolumn{2}{|c|}{ A3. DIMENSION "TECNOLOGLA" } \\
\hline \multicolumn{2}{|c|}{ Subdimension: Infraestructura de banda ancha } \\
\hline Indicador 6 & Nümero de accesos de banda ancha \\
\hline \multicolumn{2}{|c|}{ Subdimensión: Demanda tecnológica } \\
\hline Indicador 7 & Número de líneas de banda ancha por habitante \\
\hline \multicolumn{2}{|c|}{ A4. DIMENSION "TOLERANCIA, DIVERSDAD Y APERTURA" } \\
\hline Indicador 8 & Porcentaje de población extranjera \\
\hline Indicador 9 & Porcentaje de población que reside en el mismo municipio de nacimiento \\
\hline Indicador 10 & Nümero de estudiantes universitarios extranjeros \\
\hline Indicador 11 & Número de asodaciones de carácter social \\
\hline Indicador 12 & Relación entre $\mathrm{n}^{\circ}$ de matrimonios homosexuales y heter osexuales \\
\hline Indicador 13 & Diversidad religiosa \\
\hline \multicolumn{2}{|c|}{ A5. DIMENSION "TALENTO" } \\
\hline \multicolumn{2}{|c|}{ Subdimensión: Capital humano } \\
\hline Indicador 14 & $\begin{array}{l}\text { Nümero de egresados universitarios y grado de inserción en estudios rela cion ados } \\
\text { con las industrias culturales y creativas. }\end{array}$ \\
\hline Indicador 15 & Número de investigadores \\
\hline \multicolumn{2}{|c|}{ Subdimensión: Producción dentifica } \\
\hline Indicador 16 & $\begin{array}{l}\text { Nümero de publicaciones en revistas cientfficas publicadas por investigadores de } \\
\text { la universidad }\end{array}$ \\
\hline \multicolumn{2}{|c|}{ Subdimension: Proyectos I+D y emprendimiento } \\
\hline Indicador 17 & Nümero de proyectos I+D generados por la universidad \\
\hline Indicador 18 & Número de Spin Off generados por la universidad \\
\hline \multicolumn{2}{|c|}{ Subdimensión: Propiedad industrial e intelectual } \\
\hline Indicador 19 & Nümero de patentes \\
\hline Indicador 20 & Número de solicitudes de inscripción en el Registro de la Propiedad Intelectual \\
\hline \multicolumn{2}{|c|}{ Subdimensión: Premios } \\
\hline Indicador 21 & Nümero de premios obtenidos de prestigio nacional \\
\hline \multicolumn{2}{|c|}{ A6. DIMENSION "PRODUCCION CULTURAL Y CREATIVA" } \\
\hline Indicador 22 & Nümero de rodajes realizados en la dudad \\
\hline Indicador 23 & Número de publicaciones en papel \\
\hline Indicador 24 & Nümero de revistas \\
\hline Indicador 25 & Nümero de periódicos \\
\hline Indicador 26 & Nümero de medios digitales \\
\hline Indicador 27 & Nümero de emisoras de radio \\
\hline \multicolumn{2}{|c|}{ A7. DIMENSION "EXPERIENCIA CULTURAL" } \\
\hline \multicolumn{2}{|c|}{ Subdimensión: Disponibilidad cultural } \\
\hline Indicador 28 & Nümero de exposiciones no permanentes \\
\hline Indicador 29 & Número de eventos relacionados con las artes escénicas y la música \\
\hline Subdimensión & articipación cultural \\
\hline Indicador 30 & Gasto en cultura por habitante \\
\hline Indicador 31 & Porcentaje de la población que asistió a un espectáculo de danza, teatro o música \\
\hline Indicador 32 & Indice de lectura \\
\hline Indicador 33 & Espectadores de cine \\
\hline Indicador 34 & Audiencia de los medios de comunicación locales \\
\hline Indicador 35 & Número de asociaciones y fundaciones relacionadas con la cultura \\
\hline A8. DIMENS: & $\checkmark$ "MICROPRODUCTIVIDAD" \\
\hline Subdimension & ser-generated productivity \\
\hline Indicador 36 & Nümero de tweets con el hashtag de la ciudad \\
\hline Indicador 37 & Nümero de usiarios de Linkedin \\
\hline Indicador 38 & Número de videos subidos en Youtube con la etiqueta de la dudad \\
\hline Subdimension & reative user productivity \\
\hline Indicador 39 & Nümero de videos subidos en Vimeo con la etiqueta de la ciudad \\
\hline Indicador $4^{\circ}$ & Número de canales en Vimeo con la etiqueta de la ciudad \\
\hline Indicador 41 & Número de usuarios y proyectos creativos en Behance gener ados desde la ciudad \\
\hline
\end{tabular}

Fuente: Elaboración propia.

(C) Ediciones Universidad de Salamanca / CC BY - NC ND Fonseca, Journal of Communication, n. 13, 2016, pp. 129-146 


\subsection{Política cultural}

Este dominio analiza la aplicación de las políticas culturales por parte de las instituciones públicas con base en tres factores que conforman las correspondientes dimensiones. En primer lugar, la dimensión «gasto público», en la que se contempla el esfuerzo de gasto realizado por las corporaciones locales. Es necesario precisar que, aunque el gasto público en un territorio determinado lo realizan distintas administraciones como ayuntamientos, diputaciones, comunidades autónomas o la administración central, se ha limitado el análisis a las corporaciones locales, concretamente a los ayuntamientos, al tratarse de la administración más cercana al ciudadano y la que mayor esfuerzo de gasto realiza, con más del 57\% del total de las administraciones (Ministerio de Educación, Cultura y Deporte, 2015, p. 87). El gasto público en cultura aparece integrado en distintos índices, aunque de forma explícita en el Honk Kong Cretivity Index, el European Creativity Index o el CCI Creative City Index.

En la dimensión «promoción de la cultura» se agrupan indicadores referidos a la promoción de la cultura participativa, es decir, a la facilitación del uso y consumo cultural por parte de la administración local. Se distingue, por un lado, las distintas infraestructuras culturales que se ponen a disposición del ciudadano y, por otro, las diversas ayudas para la promoción de la cultura de base. En los índices analizados anteriormente, estos indicadores aparecen integrados en dimensiones como oferta cultural, financiación y políticas públicas (Picard, Gronlud y Toivonen, 2003; Hui, et al., 2004; Comisión Europea, 2009a; Hartley, et al., 2012).

Por último, en la dimensión «servicios de apoyo a las industrias culturales y creativas» se definen dos grupos de indicadores que remiten a las infraestructuras que las corporaciones locales ponen a disposición del sector y a las ayudas o incentivos que lo benefician. Ambos tipos de políticas públicas exceden al sector cultural y creativo integrándose en conceptos más genéricos como emprendimiento o innovación. Por tanto, estos indicadores cuantificarán las infraestructuras y ayudas disponibles, aunque no exclusivas, para las industrias culturales y creativas. Esta dimensión toma como referencia el apoyo público del CCI Creative City Index (Hartley, et al., 2012) y la infraestructura social y cultural del Hong Kong Creativity Index (Hui, et al., 2004).

Tabla 4. Dominio Política Cultural

\begin{tabular}{|l|l|}
\hline \multicolumn{2}{|l|}{ B1. DIMENSION “GASTO PƯBLICO” } \\
\hline Indicador 42 & Gasto por habitante de la corporación local \\
\hline Indicador 43 & Gasto publico en cultura de la corporación local \\
\hline B2. DIMENSION “PROMOCION DE LA CULTURA” \\
\hline Subdimensión: Infraestructuras para la creación y participación cultural \\
\hline Indicador 44 & Número de museos \\
\hline Indicador 45 & Número de archivos \\
\hline Indicador 46 & Número de bibliotecas \\
\hline Indicador 47 & Número de teatros \\
\hline Subdimensión: Medidas de apoyo \\
\hline Indicador 48 & Ayudas e incentivos \\
\hline Indicador 49 & Premios \\
\hline B3. DIMENSION “SERVICIOS DE APOYO A LAS INDUSTRLAS CULTURALES Y CREATIVAS” \\
\hline Indicador 50 & $\begin{array}{l}\text { Infraestructuras: espacios y equipamientos especializados, coworking, } \\
\text { mediacentres, etc. }\end{array}$ \\
\hline Indicador 51 & Ayudas a la financiación, promoción, internacionalización, etc. \\
\hline
\end{tabular}

Fuente: Elaboración propia. 


\subsection{INDUSTRIAS CULTURALES Y CREATIVAS: CREATIVE OUTPUT}

Los indicadores que miden la contribución económica del sector cultural y creativo son también recurrentes en la mayoría de índices creativos, como es el caso del European Creativity Index de la Comisión Europea (2009), el Honk Kong Creativity Index (Hui, et al., 2004) o el CCI Creative City Index de Hartley (2012). En este marco se analizan tres grupos de indicadores o dimensiones que remiten al crecimiento económico o generación de riqueza, al número de empresas que conforman el sector y al mercado laboral o empleo cultural y creativo.

Tabla 5. Dominio Industrias Culturales y Creativas: Creative Output

\begin{tabular}{|l|l|}
\hline \multicolumn{2}{|l|}{ C1. DIMENSION “RIQUEZA" } \\
\hline Indicador 52 & Facturación o ingresos de las industrias del sector \\
\hline C2. DIMENSION “TEJIDO EMPRESARIAL" \\
\hline Indicador 53 & Número de empresas culturales y creativas \\
\hline C3. DIMENSION “MERCADO LABORAL" \\
\hline Indicador 54 & $\begin{array}{l}\text { Contratos registrados en los servicios públicos de empleo en el sector cultural y } \\
\text { creativo }\end{array}$ \\
\hline Indicador 55 & $\begin{array}{l}\text { Paro registrado en los servicios públicos de empleo en el sector cultural y } \\
\text { creativo }\end{array}$ \\
\hline Indicador 56 & $\begin{array}{l}\text { Porcentaje de trabajadores por cuenta propia en relación a los trabajadores por } \\
\text { cuenta ajena }\end{array}$ \\
\hline
\end{tabular}

Fuente: Elaboración propia.

\section{DisCUSIÓN DE LOS RESUlTADOS}

En la revisión realizada se han presentado los índices que consideramos más importantes para nuestra investigación. Cada uno de ellos aporta distintos enfoques en la evaluación de la creatividad del territorio, desde la pionera y más influyente aportación de Richard Florida (2002), hasta la última y más compleja de John Hartley (2012).

Aunque cabría hablar de índices floridianos, refiriéndonos a la variedad de adaptaciones que se han hecho del Creative Cities Index de Richard Florida, es más preciso señalar que los distintos índices han evolucionado hacia al estudio y conocimiento de la creatividad desde una perspectiva cada vez más compleja. De esta manera se añaden nuevas visiones que enfatizan la importancia de la cultura (Cultural Life Index) o las políticas públicas (Honk Kong Creativity Index). También se acaban incorporando los resultados obtenidos por las industrias creativas como dimensiones a tener en cuenta en los índices (European Creativity Index).

Pero, a lo largo de estos años, no solo se ha ampliado el campo de análisis, sino también los enfoques que sobre él convergen. En algunos modelos se pone el foco en los procesos (Global Cities Index) o se personaliza la evaluación en función de los actores intervinientes en el ecosistema creativo (Global Power Cities Index). En todos los casos, sin embargo, queda patente el esfuerzo de adaptación de los índices a la realidad evaluada, es decir, a las condiciones particulares del territorio a analizar. Esto hace que la evaluación de la creatividad sea compleja y no definitiva, con una renovación y actualización permanente de los índices.

La propuesta de índice presentada en este estudio evalúa la potencialidad de las industrias culturales y creativas en un territorio determinado, concretamente en ciudades medianas y pequeñas, permitiéndonos identificar los elementos que contribuyen al desarrollo de las industrias culturales y creativas en estos entornos urbanos. Se trata, pues, de un índice creado ad hoc con el objetivo de describir y conocer una realidad concreta. Dentro del índice se pueden encontrar indicadores estándar, ya conso- 
lidados y utilizados en otros índices, y otros más novedosos que complementan a los primeros, analizando nuevas dinámicas y tendencias.

El valor añadido que ofrece nuestro índice radica en el análisis de las interrelaciones entre la base cultural y creativa de un territorio, las políticas culturales que se aplican en él y el tejido productivo cultural y creativo de ese entorno. En definitiva, se trata de una herramienta de análisis desde la perspectiva cultural, social y económica que pone especial énfasis en la contribución del ciudadano creativo y en la estrategia de las administraciones públicas en materia cultural con la mirada puesta en el crecimiento económico.

\section{CONCLUSIONES}

Las industrias culturales y creativas constituyen un sector de primera importancia, estratégico, en la economía del conocimiento. De ahí que, para conocer con fundamento las razones en las que descansa su desarrollo, se estén elaborando desde hace algunos años una serie de herramientas en los que apoyar las políticas públicas.

A estos efectos, planteamos el índice de potencialidad de las industrias culturales y creativas, una herramienta construida a partir del estudio y del análisis de los índices de creatividad existentes, con el objetivo principal de evaluar la capacidad de desarrollo económico del sector cultural y creativo en un territorio con base en tres vectores que interactúan: la base cultural y creativa, la política cultural y las industrias culturales y creativas. La actualización de este índice, la validación de sus elementos y su adecuación a la realidad evaluada se preserva, entre otras formas, gracias a la utilización de grupos de expertos.

A modo de ensayo, se ha llevado a cabo la aplicación práctica del índice mediante un estudio exhaustivo de las mayores ciudades de Andalucía, extrayéndose conclusiones que enfatizan la importancia de las políticas públicas en el sector como catalizadoras de la base cultural y del sector industrial cultural y creativo (Castro-Higueras, 2015). Este primer trabajo de campo pretende ser el inicio de posteriores investigaciones en las que se ampliarán sus resultados mediante la utilización de muestras de mayor tamaño y estudios longitudinales que analicen su evolución en el tiempo.

\section{REFERENCIAS BIBLIOGRÁFICAS}

Acs, Z. J., \& Megyesi, M. I. (2009). Creativity and industrial cities: A case study of Baltimore. Entrepreneurship and Regional Development, 21(4), 421-439.

AT Kearney. (2014). Global cities index and emerging cities outlook. Recuperado de: https://www.atkearney.com/documents/10192/4461492/Global+Cities+Present+and+Future -GCI+2014.pdf/3628fd7d-70be-41bf-99d6-4c8eaf984cd5

Batthyány, K., Cabrera, M., Alesina, L., Picasso, F., Ramírez, J., \& Rojo, V. (2011). Metodología de la investigación en ciencias sociales. Montevideo: Universidad de la República.

Boschma, R. A., \& Fritsch, M. (2009). Creative class and regional growth: Empirical evidence from seven european countries. Economic Geography, 85(4), 391-423.

Bowen, H. P., Moesen, W., \& Sleuwaegen, L. (2008). A composite index of the creative economy. Review of Business and Economics, 53(4), 375-397.

Bustamante, E. (2011). La creatividad ¿contra la cultura? Las industrias creativas: Amenazas sobre la cultura digital (pp. 15-19). Barcelona: Gedisa.

Castro-Higueras, A. (2015). Industrias culturales y creativas y su índice de potencialidad. Las ciudades de Sevilla, Málaga y Córdoba como casos de estudio (Tesis doctoral). Universidad de Málaga, Málaga. 
Clark, T. N. (2004). Urban amenities: Lakes, opera, and juice bars: Do they drive development? Research and Urban Policy, 9, 103-140.

Comisión Europea. (2006). Economy of culture in Europe. Bruselas: Kea European Affairs. Recuperado de http://ec.europa.eu/culture/library/studies/cultural-economy_en.pdf

Comisión Europea. (2009a). The impact of culture on creativity. Recuperado de http://www.keanet.eu/docs/impactculturecreativityfull.pdf

Comisión Europea. (2009b). Innovation union scoreboard. Recuperado de http://bookshop.europa.eu/en/european-innovation-scoreboard-2008pbNBNA23728/?CatalogCategoryID=Gj0KABst5F4AAAEjsZAY4e5L

Comisión Europea. (2010). Agenda Digital para Europa. Recuperado de http://eur-lex.europa.eu/legalcontent/ES/TXT/PDF/?uri=CELEX:52010DC0245\&from=ES

Comisión Europea. (2015). Innovation union scoreboard. Recuperado de http://ec.europa.eu/growth/industry/innovation/facts-figures/scoreboards/files/ius2015 en.pdf

Department for Culture, Media and Sports (DCMS). (2010). Creative economy programme - working group reports. Recuperado de

http://webarchive.nationalarchives.gov.uk/+/http:/www.culture.gov.uk/images/publications/c epworkinggroupreports.pdf

Florida, R. (2002). The rise of the creative class: And how it's transforming work, leisure, community and everyday life. New York: Basic Books.

Florida, R., \& I. Tinagli. (2004). Europe in the creative age. New York: Demos.

Florida, R., Mellander, C., Stolarick, K., Sik, K., Matheson, Z \& Hopgood, M. (2011). Creativity and prosperity: The global creativity index. Toronto: Martin Prosperity Institute.

Glaeser, E. L., \& Saiz, A. (2004). The Rise of the Skilled City. Brookings-Wharton Papers on Urban Affairs, $5,47-94$.

Gobierno de España (2013). Agenda Digital Para España. Recuperado de http://www.agendadigital.gob.es/Paginas/Index.aspx

Hagel, J., Brown, J. S., \& Davison, L. (2009). The shift index: Uncovering the emerging logic of deep change. New York: Deloitte.

Hartley, J., Potts, J., MacDonald, T., Erkunt, C., \& Kufleitner, C. (2012). Creative city index - final report. Queensland: ARC Centre of Excellence for Creative Industries and Innovation.

Hernández Sampieri, R., Fernández Collado, C. \& Baptista Lucio, P. (1998). Metodología de la investigación. McGraw-Hill.

Hollanders, H., \& Van Cruysen, A. (2009). Design, creativity and innovation: A scoreboard approach. Pro Inno Europe, Inno Metrics: Holanda.

Hui, D., Ng, C., Mok, P., Ngai, F., Wan-kan, C., \& Yuen, C. (2005). A study on creativity index. Hong Kong: Home Affairs Bureau.

Jacobs, J. (1961). The death and life of great american cities. New York: Random House LLC.

Konow, I., \& Pérez, G. (1990). Métodos y técnicas de investigación prospectiva para la toma de decisiones. Santiago de Chile: Universidad De Chile.

Landeta, J., Matey, J., Ruiz, V., \& Villarreal, O. (2002). Alimentación de modelos cuantitativos con información subjetiva: Aplicación delphi en la elaboración de un modelo de imputación del gasto turístico individual en catalunya. Qüestiió, 26(1-2), 175-196.

Landry, C. (2010). Índice de creatividad de bilbao biækeaia. Bilbao: BM30. Recuperado de http://www.bm30.es/Indice_Creatividad_Bizkaia.pdf 
Landry, C., \& Hyams, J. (2012). The creative city index: Measuring the creative pulse of your city. London: Comedia.

Marlet, G., \& Van Woerkens, C. (2004). Skills and creativity in a cross-section of dutch cities. Tjalling C.Koopmans Institute Discussion Paper Series, 4(29).

Miller, T. (2009). Can natural luddites make things explode or travel faster? the new humanities, cultural policy studies, and creative industries. En J, Holt \& A. Perren (Eds.), Media Industries: History, Theory, and Method (pp. 184-198). Malden MA: Wiley-Blackwell.

Ministerio de Educación, Cultura y Deporte. (2015). Anuario de estadísticas culturales 2013. Recuperado de http://www.mecd.gob.es/servicios-al-ciudadano-mecd/dms/mecd/servicios-al-ciudadanomecd/estadisticas/cultura/mc/naec/2015/Anuario de Estadisticas Culturales 2015.pdf

Mori Memorial Foundation. (2014). Global power city index. Tokio: Institute for Urban Strategies.

Nardo, M., Saisana, M., Saltelli, A., Tarantola, S., Giovannini, E., \& Hoffmann, A. (2008). Handbook on constructing composite indicators: Methodoloy and user guide. Paris: Organisation for Economic Cooperation and Development. OECD.

Oliveira, C. M. (2012). Creative indexes: Economic space matters? Unpublished Master, Universidade de Porto, Porto.

Organización para la Cooperación y el Desarrollo Económicos (OCDE). (2013). Science, technology and industry scoreboard 2013. Paris: OECD.

Peck, J. (2005). Struggling with the creative class. International Journal of Urban and Regional Research, 29(4), 740-770.

Picard, R. G., Grönlund, M., \& Toivonen, T. (2003). Means for overall assessment of cultural life and measuring the involvement of the cultural sector in the information society. Helsinki: Ministry of Education.

Sánchez Fernández, G. (2009). Análisis de la sostenibilidad agraria mediante indicadores sintéticos: Aplicación empirica para sistemas agrarios. Madrid: Universidad Politécnica.

Schlesinger, P. (2009). Creativity and the experts new labour, think tanks, and the policy process. The International Journal of press/politics, 14(1), 3-20.

Schwab, K., \& Sala-i-Martin, X. (2011). The global competitiveness report 2011-2012. Geneva: World Economic Forum.

United Nation Conference on Trade and Development (UNCTAD). (2013). Creative economy report 2013. Recuperado de http://unctad.org/es/Docs/ditctab20103 sp.pdf

United Nations Educational, Scientific and Cultural Organization (UNESCO). (2010). Políticas para la creatividad. guia para el desarrollo de las industrias culturales. Recuperado de http://www.unesco.org/new/fileadmin/MULTIMEDIA/HQ/CLT/images/UNESCOCultural andCreativeIndustriesguide 01.pdf

Zallo, R. (1988). Economía de la comunicación y la cultura. Madrid: Ediciones Akal.

Zallo, R. (2011). Industrial culturales y territorios creativos. Los límites de la transversalidad. Las industrias creativas: Amenazas sobre la cultura digital (pp. 153-187). Barcelona: Gedisa. 